\title{
Egypte: Les ONG doivent unir leurs forces pour éradiquer l'excision
}

Frontiers in Reproductive Health

Follow this and additional works at: https://knowledgecommons.popcouncil.org/departments_sbsr-rh

Part of the Public Health Education and Promotion Commons, and the Women's Health Commons How does access to this work benefit you? Let us know!

\section{Recommended Citation}

"Egypte: Les ONG doivent unir leurs forces pour éradiquer l'excision," FRONTIERES Résumés de Recherche Opérationnelle. Washington, DC: Population Council, 2000. 


\title{
Egypte Les ONG doivent unir leurs forces pour Excision éradiquer l'excision
}

$N^{\circ} 14$

\begin{abstract}
En Egypte, quinze ONG nationales sont activement impliquées dans la mise en ouvre des programmes visant à éradiquer la pratique de l'excision. Pour que ces programmes soient plus efficaces, les ONG doivent former une coalition, faire du plaidoyer, former les membres en communication, et évaluer l'impact de ces programmes.
\end{abstract}

\section{Contexte}

Tout récemment, la pratique de l'excision était presque généralisée en Egypte. Toutefois, une étude nationale menée en 1998 a montré les premiers signes du déclin de cette pratique à partir de 1994 parmi les adolescentes (ElGibaly et al., 1999).

Les ONGs travaillant dans les domaines du développement communautaire, de la santé et des droits de la femme ont joué un rôle de leader dans les activités de plaidoyer pour l'éradication de la pratique de l'excision en Egypte. Le Population Council a effectué d'Août 1999 à Février 2000 une étude pour documenter et évaluer l'impact des programmes d'éradication de la pratique de l'excision. Pour identifier les ONG qui étaient les plus activement impliquées dans les programmes d'éradication de la pratique de l'excision, les chercheurs ont eu à mener des entretiens téléphoniques. Ensuite, ils ont eu des interviews approfondis avec 15 représentants d'ONG, le personnel du Ministère de la Santé et de la Population, de l'UNICEF et du Fonds des Nations Unies pour la Population (FNUAP).

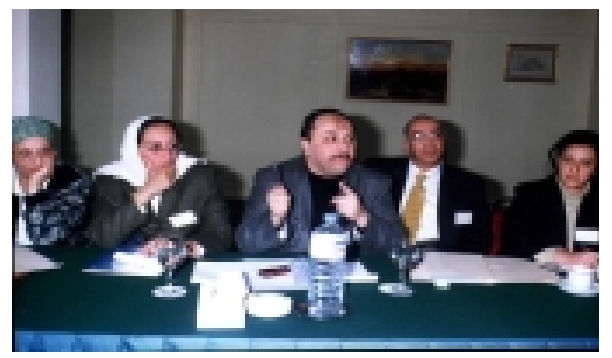

\section{Résultats}

La plupart des ONG n'ont mis en place aucun mécanisme d'évaluation de l'impact de leurs interventions. Certaines ONG collectent des informations sur les indicateurs de processus, tel que le nombre de réunions organisées, le nombre de participants et de demandes d'informations. Peu d'ONG mesurent l'impact de leurs interventions sur les connaissances, les attitudes et les pratiques des participants.

Chacun des quatre modèles d'intervention de base, identifiés comme outils d'évaluation, a servi à aborder quelques aspects du processus de changement de comportement, allant de la sensibilisation à l'acquisition des connaissances, en passant par les discussions sur la pratique de l'excision; tout cela en vue de la prise de position ferme contre cette même pratique. Ces quatre modèles d'interventions sont les suivants :

\& La sensibilisation. Beaucoup d'ONG organisent de grandes conférences et séminaires pour les populations, avec la participation des médecins et des leaders religieux, pour discuter des méfaits de la pratique de l'excision. Ces forums permettent de réunir un grand nombre de personnes à un coût relativement bas. 


\section{\& Faire des membres de la communauté des Implications pour les programmes}

\section{agents de changement. Quelques ONG}

forment des membres influents de la communauté ou des individus opposés à la pratique de l'excision (des déviants positifs) dans le but de sensibiliser les autres membres de leur communauté. Les leaders des ONG ont témoigné que cette approche permettait d'acquérir plus de connaissances et d'avoir des changements de comportement face à la pratique de l'excision.

\& Développement communautaire. Plusieurs ONG ont intégrés des messages contre la pratique de l'excision dans les cours d'alphabétisation et dans un programme pour jeunes handicapés. Quelques ONG ont ajouté une composante sur la lutte contre l'excision dans tous leurs programmes de développement. Cette approche est prometteuse, puisqu'elle cible toute la communauté et touche les populations à travers des voies multiples : les séminaires, les visites à domicile et les cours d'alphabétisation. Toutefois, l'approche est coûteuse et exige des efforts considérables.

\section{\& Plaidoyer. Quelques ONG se sont engagées} dans des activités de plaidoyer telles que : l'organisation de réunions de sensibilisation pour les membres du gouvernement ; l'information des décideurs politiques, des chercheurs et des journalistes ; la formation de coalitions avec les autres ONG; le développement des aptitudes des leaders locaux en plaidoyer ; la production d'émissions audiovisuelles sur l'excision.

\section{La plupart des représentants des ONG} reconnaissent l'importance des réseaux et des coalitions de lutte contre la pratique de l'excision. Seulement deux d'entre elles sont effectivement affiliées à ces groupes.
Au cours d'un séminaire de deux jours qui s'est tenu en janvier 2000 au Caire, quarante représentants d'ONG, d'agences gouvernementales, d'instituts de recherche, de bailleurs de fonds et de partenaires au développement ont eu à discuter des résultats de l'évaluation et ont fait les recommandations suivantes :

- Les ONG doivent former des coalitions pour plus de synergie et de complémentarité dans leurs actions respectives. Elles doivent impliquer les agences gouvernementales, les média, les institutions de recherche et les communautés dans des interventions à grande échelle.

- Il faut davantage des activités de plaidoyer, plus particulièrement celles associant les médias et les autorités politiques en vue de créer un environnement sociopolitique hostile à la pratique de l'excision en Egypte.

Les messages contre la pratique de l'excision doivent mettre l'accent sur les aspects sociaux, religieux et légaux du problème, au lieu de s'appesantir sur les risques d'ordre sanitaire.

- Les ONG doivent développer des partenariats avec les instituts de recherches pour bénéficier d'une assistance technique dans leurs activités d'évaluation. Elles doivent développer des indicateurs pour mesurer les différentes étapes du changement d'attitudes et de comportement.

- Les assistants sociaux et les animateurs de base doivent être formés aux techniques de communication et de résolution des problèmes. Ils doivent également bénéficier d'une assistance dans la définition et le suivi de leurs activités.

Septembre 2000

Abdel-Tawab, Nahla, et Sahar Hegazi.2000. « Analyse Critique des Interventions de lutte contre l'excision en Egypte ». Caire : Population Council. Pour plus d'informations ou pour obtenir une copie de la version Anglaise du Rapport Final et la version Arabe de la synthèse du Résumé de cette étude, contacter : Population Council, 6A Mohamed Bahie Eddine Barakat St., 10 ème étage, Giza, Egypte. Tél. : 202-571-9252 ; Fax:202-570-1804 ; E-mail : frontiers@pccairo.org.

Ce projet a été effectué avec l'appui de l'AGENCE AMERICAINE POUR LE DEVELOPPEMENT INTERNATIONAL (USAID) sous l'Accord de Coopération Numéro HRN-A-00-98-00012-00 